\title{
Casa Asistida, un lugar para habitar facilitador de la inclusión social para pacientes de larga institucionalización ${ }^{1}$
}

\section{Assisted House, a place to live facilitator of social inclusion for long-term institutionalized patients}

\author{
Cecilia del Carmen Molina ${ }^{2}$
}

\section{Resumen}

El presente trabajo analiza el proceso de constitución del dispositivo sustitutivo Casa de Medio Camino en su modalidad de Vivienda Asistida desde un Centro Regional de Salud Mental Público de la provincia de Santa Fe, Argentina. Para ello se focalizará en la problemática de los efectos de la institucionalización en pacientes con padecimientos subjetivos de larga internación en un hospital monovalente: ¿Cómo superarlos durante los procesos de inclusión social consecuentes a las políticas de desmanicomialización? Realizaremos, primero, una aproximación a dos procesos de desinstitucionalización internacionales, el italiano y el francés, enfatizando los dispositivos residenciales y los aportes que proponen, para luego compararlo con el dispositivo local. Se tomará como marcos normativos de las casas de convivencia, antecedentes de leyes en ésta área hasta arribar a la sanción de la primera Ley Nacional de Salud Mental No 26.557 (a partir de ahora LNSM).

Finalmente, se analizarán las particularida- des del proceso de constitución de la casa de medio camino local y la desinstitucionalización de cuatro pacientes. En las conclusiones se enunciarán los aportes, alcances y características instituyentes de dicho proceso.

Palabras claves: Casa Asistida - Departamentos terapéuticos (DT) - Vivienda de bajo alquiler (HLM) - Ley Nacional de Salud Mental No 26.657 - Ley Provincial de Salud Mental No 10.772 - Lógicas manicomiales Desinstitucionalización - Inclusión social.

\section{Summary}

The present paper analyzes the process of constitution of the alternative device Casa de Medio Camino in its modality of Assisted Housing from a Regional Center of Public Mental Health of the province of Santa Fe, Argentina. To this end, it will focus on the problems of the effects of institutionalization in patients with long-term subjective suffering in a monovalent hospital: how to overcome them during the processes of so-

\footnotetext{
${ }^{1}$ El presente artículo fue reescrito sobre la base del Trabajo Integrador Final de la Carrera de Especialización en Psicología Clínica, Institucional y Comunitaria (cohorte 2010-2011) “Casa Asistida, un espacio para habitar facilitador de la inclusión social para pacientes de larga institucionalización” (año 2014) del que soy autora y fuera dirigido por el Psicólogo y Especialista en Psicología Clínica, Institucional y Comunitaria Claudio Cúneo. ${ }^{2}$ Psicóloga, Especialista en Psicología Clínica, Institucional y Comunitaria. Trabaja en Ministerio de Desarrollo Social de la Provincia de Santa Fe, Argentina. Correo electrónico: molcecilia@hotmail.com
} 
cial inclusion consistent with the policies of de-asylum? We will make, first, an approximation to two international deinstitutionalization processes, the Italian and the French, emphasizing the residential devices and the contributions they propose. We then compare it with the local device. It will be taken as normative frameworks of the houses of coexistence, antecedents of laws in this area until arriving to the sanction of the first National Law of Mental Health N 26.557 (LNSM). Finally, the particularities of the process of constitution of the local halfway house and the deinstitutionalization of four patients will be analyzed. The conclusions will set out the contributions, scope and institutional characteristics of this process.

Keywords: Assisted Home - Therapeutic Departments (TD) - Low-rent Housing (HLM) - National Mental Health Law $N^{\circ}$ 26.657 - Provincial Mental Health Law No 10.772 - Psychiatric logics - Deinstitutionalization - Social inclusion

\section{Introducción}

En este escrito abordaremos la problemática de los efectos de la institucionalización en pacientes con padecimientos subjetivos de larga internación en hospitales monovalentes públicos y la interpelación en cuanto a: ¿cómo superarlos durante los procesos de inclusión social consecuentes a las políticas de desmanicomialización? Enmarcado en la temática del dispositivo de casa de medio camino, nos interesa, particularmente, describir y analizar cómo se fue constituyendo la primera Casa Asistida de Mujeres, creada por actores institucionales desde el mismo efector público del cual se externaron. Dentro de este recorte, se intentará profundizar en lo particular de los procesos que se fueron dando y posibilitaron hacer efectiva la desinstitucionalización de las pacientes allí alojadas.

El Centro Regional de Salud Mental (C.R.S.M.) es un hospital monovalente, con internación en salud mental y guardia permanente. Es el único efector público en la región de esta especialidad y con estas características. Dispone de dispositivos de internación para las situaciones de crisis, sin embargo, más del $50 \%$ de sus camas están ocupadas con pacientes de larga institucionalización (crónicos). ${ }^{3}$

Es desde el marco de la Ley Provincial de Salud Mental No 10.772 -sancionada en 1991 y reglamentada en 2007-, que se impulsó la transformación del C.R.S.M. y la creación de los nuevos dispositivos sustitutivos de las lógicas manicomiales. Las Casas de Medio Camino, en su modalidad de Vivienda Asistida, son uno de los proyectos surgidos en estas coyunturas. Sin embargo, no es menor y debe destacarse, la demora transcurrida desde la sanción de la ley provincial en Santa Fe hasta la creación de la primera casa en el año 2009. En este sentido, la puesta en marcha de la primera Casa Asistida desde el C.R.S.M. -en la actualidad son dos, una de mujeres y otra de varones- constituye una experiencia pionera como modo de lograr la desinstitucionalización de pacientes crónicos desde el hospital, proceso pionero en cuanto a la construcción conjunta entre usuarios y trabajadores de la salud mental que tiene por meta la tan deseada desmanicomialización. Se trata de una construcción que se renueva día a día a través de la revisión y la reformulación tanto de las prácticas de los equipos de salud como del modo de habitar de las moradoras.

A pesar de la transformación que fue atravesando el C.R.S.M., los pacientes con larga institucionalización o con repetición reitera-

\footnotetext{
${ }^{3}$ Año en el que fue realizado el TIF (2014) con las entrevistas y el material disponible hasta ese momento.
} 
das de internaciones, están atravesados por la impronta de la institución, huellas de lo que Goffman (1972) postula como institución total: los individuos tienden a permanecen apartados de la sociedad y comparten una serie de rutinas diarias administradas formalmente. En estas instituciones todos los aspectos de la vida de un individuo se desarrollan mayormente en un mismo lugar, todas las actividades diarias se llevan a término en compañía de los otros: están programadas y son mayormente obligatorias y se integran en un único plan racional concebido para alcanzar los objetivos de la institución. "La institución controla el tiempo del interno e invade su sentido de identidad originando la mortificación del yo". (Goffman, 1972, p.27). Desde el momento del ingreso se priva al paciente de las posibilidades y de los objetos que definen su identidad, pierde privacidad.

Para el presente trabajo partimos de considerar que la cronicidad hospitalaria es el resultado de largos períodos de internación y que las consecuencias que produce en los pacientes son, en su mayoría, devastadoras. Las más importantes a reseñar son: "el distanciamiento del entorno familiar, las pérdidas de capacidades laborales y de los hábitos de la vida cotidiana, la dificultad en el cuidado de sí mismo debido a la relación de dependencia establecida con la institución, escollos para hacer lazo fuera del hospital". (Scandizzi, 2013)

De ahí la importancia de conocer e indagar el dispositivo Casa Asistida, de evaluar los alcances del proyecto en cuanto a la búsqueda del mantenimiento o recobro de los lazos sociales en la comunidad y de los hábitos del cuidado de sí, de propiciar la continuidad o el reencuentro con los lazos familiares y de amistad. Este dispositivo sustitutivo se desarrolla en el marco de las políticas de desinstitucionalización promovida por las leyes, identificando los aspectos fundamentales del marco legal delineado por las leyes provinciales y nacionales de salud mental en la Argentina.

En cuanto a la metodología utilizada se recurrió a la construcción de un marco teórico general respecto de experiencias de desinstitucionalización internacionales que nos preceden y revisión del marco legal de salud mental en la Argentina. Con respecto al material de campo, se basó en material resultado de entrevistas semiestructuradas y abiertas a los principales actores que participaron del proyecto y proceso de constitución del nuevo dispositivo. Por último y con la finalidad de integrar las informaciones específicas, se asistió a conferencias sobre la temática y la realización de observaciones tanto del C.R.S.M. como de la residencia analizada.

\section{Experiencia italiana}

Entre las experiencias de desinstitucionalización a nivel mundial, la experiencia italiana sobre la reforma psiquiátrica y sus procesos de desinstitucionalización, ha marcado un profundo cambio y una notable influencia en el resto del continente. Fue un proceso de profunda transformación que partió desde los manicomios hasta lograr servicios de salud mental en el territorio. En el año 1978, la ley de reforma de la psiquiatría, más conocida como Ley 180 o ley Basaglia puso en movimiento un proceso nacional italiano de eliminación de los hospitales psiquiátricos. Esta ley permitió el cierre definitivo de dichas instituciones recién al final de la década del noventa. Asimismo, la creciente instauración de servicios basados en el territorio facilitó a las personas con sufrimientos mentales vivir $y$ desenvolverse normalmente en un contexto social.

Luego de haber sido director del hospital local de Gorizia, Franco Basaglia toma la 
dirección del hospital psiquiátrico provincial de Trieste en 1971. Ya en Gorizia descubre el tratamiento "carcelario" que recibían los pacientes internados y los dolorosos procedimientos de muy dudosa eficacia a los que eran sometidos, lo que contribuyó a consolidar su compromiso con la democratización de la psiquiatría, la humanización de sus métodos y procedimientos.

En el libro La institución negada. Informe de un Hospital Psiquiátrico, Basaglia (1972) denuncia que los hospitales psiquiátricos no podían ser modificados ya que, obedeciendo a normas $y$ leyes de orden público y de control social, no podían cumplir con los objetivos de asistencia y de rehabilitación, constituyéndose más bien en productores de enfermedad. La idea que se imponía con fuerza era ir más allá del manicomio, es decir, transformar su organización no tanto para reformarla, sino para superarla.

En relación a los cambios legislativos que acompañaron a la reforma italiana, cabe mencionar que cuando asume Basaglia como director del hospital de Trieste, la atención psiquiátrica estaba regulada por una ley que databa de 1904 cuyo eje era la protección de la sociedad de la peligrosidad de los enfermos mentales. Lo principal era la custodia y no la atención, las personas que se internaban perdían sus derechos civiles. En aquel entonces los manicomios estaban a cargo de administraciones provinciales. La ley 180 comienza una nueva etapa de regulaciones normativas de los marcos nacionales y provinciales. Asimismo, define responsabilidades y funcionamiento de los departamentos de Salud Mental. Entre los aspectos más característicos que implica esta ley, cabe destacar la eliminación de los hospitales psiquiátricos y la restitución del derecho de las personas al tratamiento fundado en la atención y en la salud, y no ya en el criterio de peligrosidad.

La experiencia del hospital de Trieste fue pionera y paradigmática de los procesos que posteriormente sucedieron en Italia como así también en nuestro país.

¿En qué consistió aquella experiencia? El equipo de trabajo comenzó a usar normativas de hospitalización voluntaria, sin privar a los pacientes de sus derechos, acrecentando la posibilidad de movimiento dentro y fuera de la institución. Para lograrlo, los trabajadores reorganizaron profundamente los espacios internos y la relación entre los pacientes y el personal. Se conformaron cinco equipos que pasaron a encargarse de cinco áreas de la ciudad, mientras se llevaban a cabo reuniones diarias de la dirección, de los equipos, y del personal, como espacios de reflexión y discusión. De la misma forma en las asambleas con los pacientes, coordinadas por Basaglia, se debatía sobre el devenir de los cambios. Conjuntamente, se eliminó el electro shock y todas las medidas de contención física, se abrieron las puertas del hospicio y se crearon espacios mixtos de hombres y mujeres. Por otra parte, cobraban fuerza los espacios de vida comunitaria en el hospital como las fiestas, periódicos de autogestión y bares. El trabajo terapéutico se basó en la reconstrucción de las historias de vida de los pacientes, en la tarea de restituir vínculos con las familias y con sus lugares de orígenes.

Dos aspectos son importantes a destacar de este proceso. Por un lado, a medida que los espacios del hospital se reorganizaban, se instituyeron grupos de convivencia, primero, dentro de la estructura del predio y, luego, en la ciudad. Por otro lado, se constituyeron formas de organización de los pacientes que incluyeron la creación, en 1972, de la Cooperativa de Obreros Unidos. En ella 60 personas internadas trabajan en la limpieza de salas, cocina y parque, a través de un contrato sindical con el que cada interno pasó a ser, también, un trabajador ya que recibían un salario. Los cambios mencionados demostraron que no era la enfermedad la que impedía construir otros modos de 
rehabilitación, sino las normativas jurídicas y administrativas de exclusión de los pacientes.

A continuación, se desarrollará brevemente el dispositivo italiano de los departamentos terapéuticos, para luego retomarlo cuando se analice la experiencia de la Casa Asistida en la Argentina.

La experiencia en Italia de los Departamentos Terapéuticos (DT) nació en los años setenta y fueron las primeras señales concretas de superación del Hospital Psiquiátrico; un proceso que se consolidó en los años noventa, precisamente con la constitución de los DT bajo el nombre GA -gruppi apartamento(2013, Ricerca del Dipartimento Interaziendale di Collegno / ASO - S. Luigi Gonzaga -Universitario di Salute Mental - Direttore: Prof. Pier Maria Furlan).

En el año 1995 se dieron las primeras directrices para pensar la superación del hospital manicomial y la creación de las estructuras residenciales. Específicamente, nos detendremos en el modelo de residencia de la región de Piamonte, que a través de los Decretos 357/370 del 28 de enero de 1997 establece y posibilita las intervenciones alternativas a la internación y reinserción de los pacientes en estructuras residenciales protegidas a través de los Departamentos Terapéuticos.

¿En qué consisten los DT? Los Departamentos Terapéuticos son soluciones habitacionales para responder a exigencias específicas de residencia asistida de tipo no asilar, dirigida a pacientes que han alcanzado una fase avanzada de su reinserción social. Los DT pueden concentrarse en grupos habitacionales cada uno de los cuales puede alojar como máximo cinco pacientes. Cada Grupo de DT no puede tener más de dos núcleos habitacionales gestionados o bien directamente por el Departamento de Salud Mental -que garantiza un soporte domiciliario con recursos propios o tercerizados- o bien, por asociaciones de familiares o voluntarios. Los niveles de asistencia y protección de cada Grupo de Departamento Terapéutico varían en función de las características de los moradores y de los proyectos terapéuticos grupales e individuales. Los usuarios contribuyen económicamente a la gestión de los mismos. A cargo del Sistema Sanitario Nacional sólo quedan los costos de las actividades sanitarias.

Los Grupos de DT representan exclusivamente una unidad habitacional y los pacientes quedan a cargo del departamento de Salud Mental que compete por territorio de residencia, el cual es directamente responsable del proyecto terapéutico de rehabilitación. En cuanto a la estrategia terapéutica de rehabilitación que sustenta a los DT, se focaliza en una forma de residencia que tiene en cuenta "lo pequeño", es decir, las situaciones de convivencia entre pocas personas, en contraposición con los grandes números de las estructuras protegidas, lo cual permite examinar de modo analítico y organizado la problemática de la casa.

\section{La experiencia francesa}

En el presente apartado se presentarán los postulados básicos de la Psiquiatría de Sector en Francia que, denunciando la crisis de la asistencia psiquiatría tradicional en su papel exclusivamente tutelar y no terapéutico (asilos), produce una relativización del saber psiquiátrico, y junto a ello la inevitable necesidad de redefinir el papel del psiquiatra. Luego se puntualizará sobre la vivienda de bajo alquiler (habitation a loyer moderé o HLM) francesa. La inclusión de su descripción y análisis se debe a que, en tanto viviendas sociales, en Francia, cumplen la función de casas de medio camino sin asistencia.

Siguiendo la propuesta de Cúneo (2013) sobre el proceso de desinstitucionalización 
francesa, cabe señalar el impacto que la Segunda Guerra Mundial produjo en la opinión pública, generando una importante sensibilización con respecto a las condiciones en que se encontraban los enfermos mentales en los hospitales. La muerte de varios de ellos debido a limitaciones alimenticias durante el combate dio lugar a reiteradas comparaciones entre los manicomios y los campos de concentración. Se inicia así una profunda crítica hacia el sistema asilar, sostenida a partir de 1944 por un movimiento alternativo al manicomio, protagonizado al comienzo por psiquiatras marxistas y representantes de la doctrina "post esquiroliana" denominado así por Lucien Bonnafé. El post esquirolismo procuraba superar el manicomio, postulaba una visión más integral del tratamiento de la locura que no tuviera como eje al manicomio tradicional.

En marzo de 1960, ciertos triunfos políticos y normativos de la doctrina post esquiroliana se tradujeron en la aparición de una circular del Ministerio de Salud Pública, que establece un programa de organización y equipamiento de los departamentos en materia de tratamiento de las enfermedades mentales. El principio esencial de la organización consistió en separar lo menos posible al enfermo de su familia y de su medio, asimismo se reconocía que la mayor parte de los enfermos mentales tenían problemas médico-sociales importantes que no se solucionaban por la hospitalización, y que la propia hospitalización los agravaba aún más. A través de la circular se reconoce oficialmente el fracaso del manicomio. La circular aclara que la hospitalización de un enfermo es sólo una etapa del tratamiento y que debe ser continuado en las estructuras de prevención y post cura. Para esto propone dividir el departamento en una cantidad de sectores geográficos y en cada uno de ellos, el equipo médico-social deberá asegurar a todos los pacientes, hombres y mujeres, la continuidad del tratamiento sin hospitaliza- ción, incluyendo el seguimiento post cura.

Las viviendas de bajo alquiler (habitation a loyer moderé o HLM) se trata de viviendas administradas por una institución, pública o privada, que se beneficia de una financiación pública parcial, directa (subvención) o indirecta (créditos, subsidios). Son residencias de bajo costo, también denominadas "sociales". El 20\% de la población francesa vive en viviendas de bajo alquiler. (2006 "L'accès et le maintien dans le logement des personnes ayant des difficultés de santé mentale" - L'union Sociale Pour L'habitatles Revues Paris -France-)

El hecho de que gran cantidad de personas con padecimientos psíquicos en Francia encuentre un lugar de alojamiento en este tipo de viviendas, es relevante para el análisis del dispositivo Casa de Medio Camino; ya que describe el modo de funcionamiento y normativa de las mismas.

Como consecuencia de la política masiva de deshospitalización y el cierre de camas de hospital, el acceso a la vivienda que administran los HML se vio muy demandado por distintos actores, hospitales, asociaciones, que piden que se les dé soluciones de alojamiento a personas que sufren una enfermedad psíquica.

Los organismos de las viviendas de bajo alquiler o HLM se encuentran con inquilinos que suelen presentar problemas de comportamiento crónicos que no logran resolver solos. Frente a estas situaciones los organismos se preguntan: ¿estos problemas se deben a una enfermedad mental? ¿Se trata de personas fragilizadas por sus condiciones de vida precarias? En el momento que los actores sociales se declaran sobrepasados: ¿qué actores podrían ayudar en la búsqueda de soluciones que permitan mantener el alojamiento de estas personas fragilizadas? ¿Cómo organizarse internamente e intervenir en modo pertinente para enfrentar correctamente estas situaciones? 
Los administradores sociales intervienen de distintos modos: lo más habitual es tratar de restablecer el uso pacífico de la vivienda y de garantizar el respeto del condominio. Debido a que son operadores sociales y urbanos, se encuentran implicados en el funcionamiento social del conjunto inmobiliario que administran y deben contribuir, junto a otros actores, a regular la vida colectiva. Para esto, cuentan con una Guía para la gestión de problemas de Salud Mental en HLM, que apunta a ofrecer información detallada para ayudar a los organismos a intervenir con criterio y a movilizar los actores sociales o del ámbito sanitario, que puedan ayudarlos a dar respuesta a las distintas situaciones que se van presentando. La guía aborda la cuestión de la vivienda adaptada a un público que sufre una fragilidad psíquica.

Las principales situaciones encontradas por los organismos de gestión de HLM son las siguientes:

- Problemas de vecindarios crónico. Pueden manifestarse inicialmente como molestias ocasionadas por un inquilino a su entorno -ruido, olores, etc.

- Problemas de magnitud inusual. El problema entre vecinos se manifiesta con una dimensión que no es habitual, asociada a un comportamiento extraño y que puede constituir un peligro potencial para la persona o para sus vecinos (peligro para bienes o personas, problemas de orden público). Los conceptos de "extrañeza" y de "peligro" pueden variar según las costumbres de vida colectiva y el grado de tolerancia del entorno.

- Dificultades en la relación administrador-inquilino. El administrador debe enfrentarse a veces con un comportamiento no habitual de un inquilino: pedidos extraños, ambivalentes u ob- sesivos, relaciones difíciles debidas a sentimientos de persecución, irritación extrema hacia el personal, problemas que sí inciden directamente en la relación con el administrador.

- Casos de aislamiento y de reclusión. Las administraciones encuentran, a menudo, personas que se dejan estar, se aíslan y se colocan a sí mismas en peligro, sin que los vecinos sean molestados durante un cierto tiempo.

- Casos de explotación o de riesgo o peligro de algunas personas. Los administradores se encuentran con situaciones de peligro o explotación hacia las personas frágiles o con capacidades diferentes por parte de miembros de la propia familia o de los vecinos más o menos próximos. Puede tratarse de personas generalmente aisladas que abren las puertas de su vivienda a todos.

Aun cuando el administrador no tenga las competencias necesarias para entender la realidad del sufrimiento psíquico de una persona, ni puede saber si una persona sufre o no de una enfermedad mental, sí se confronta a sus consecuencias sobre el funcionamiento social de su patrimonio. Tiene, entonces, responsabilidades y un rol que desempeñar ya sea en el largo plazo y en la urgencia. Para esto la guía señala cinco pasos que podrían ser necesarios: a) la identificación de la situación crítica puede estar relacionada con una problemática de fragilidad psicológica o de salud mental; b) evaluar la situación: se trata de analizar y de comprender el problema y decidir si se trata de algo que puede ser tratado y resuelto en el condominio y/o si tiene que ser orientado a profesionales competentes; c) es necesario que el administrador identifique y contacte los operadores competentes para que se hagan cargo de la situación y que pueda estar a la al- 
tura de proveer toda la información necesaria para su seguimiento. Frente a la persona frágil, la administración funciona como puente para tramitar el pedido de ayuda; d) La gestión a largo plazo supone construir acuerdos entre los actores que accedan a tratar de forma coherente y complementaria los diferentes aspectos de la problemática; y e) la identificación de situaciones de urgencia especificadas por el Código de Sanidad Pública (peligro para la persona, su entorno, atentado al orden público) y de solicitar la intervención de las instituciones competentes dentro del marco de los procedimientos previstos por la ley.

\section{Experiencia local}

Para el desarrollo de la experiencia de la Casa Asistida en la provincia de Santa Fe se tomará como punto de partida la creación en el año 2010 de la Dirección Nacional de Salud Mental y Adicciones. Este organismo que funcionó bajo la órbita del Ministerio de Salud de la Nación, se conformó por la necesidad de fortalecer las políticas públicas de salud mental, especialmente el cumplimiento de los derechos de las personas con padecimientos mentales o adicciones. El objetivo principal era promover y coordinar redes locales, regionales y nacionales ordenadas, según criterios de riesgo, que contemplasen el desarrollo adecuado de los recursos para la atención primaria de salud mental y de las adicciones. Junto a la innovación de esta dirección, se sanciona en noviembre de 2010 la primera Ley Nacional de Salud Mental No 26.657.

Siguiendo las conceptualizaciones que señala de Galende (2011) esta ley:

"Reordena de manera profunda la relación represiva y discriminatoria del enfermo mental con el sistema judicial del Estado. Esta Ley, bajo una perspectiva de derechos humanos y de ciudadanía, y que impone la obligación del Estado de atender con protección de derechos a quienes padecen de trastorno mental, inaugura una nueva época en el modo de comprensión del Estado sobre la persona con trastorno mental y genera un nuevo marco normativo para su tratamiento". (Galende, 2011, p.10)

La reglamentación de la ley nacional es sancionada en mayo de 2013 mediante el Decreto $\mathrm{N}^{\circ} 603$ que establece la protección de los derechos de las personas con padecimiento mental en todo el territorio de nuestro país. Finalmente, en octubre de 2013, el Ministerio de Salud presenta el Plan Nacional de Salud Mental en cuyo prólogo subraya "por una salud mental para la inclusión." (Resolución 2177/2013, Plan Nacional de Salud Mental. B.O. del Ministerio de Salud de la Nación. Buenos Aires, Argentina).

\section{Casa de Medio Camino. Marco legal}

Respecto a los antecedentes normativos de la LNSM No 26.657, nuestra Ley Provincial de Salud Mental No 10.772 ya consideraba al dispositivo de la Casa de Medio Camino como un servicio alternativo. ${ }^{4}$ Así, en el artículo $18^{\circ}$ y su reglamentación dispone:

"La transformación de los Hospitales
Psiquiátricos tendrá como objetivo la
erradicación de las lógicas manicomia-
les: Medicalización de los padecimien-
tos subjetivos, internación por tiempo

${ }^{4}$ Los conceptos de alternativos y sustitutivos se tomarán como equivalentes. 
indeterminado, aislamiento social, silenciamiento, ausencia de estrategias terapéuticas complejas y singularizadas, perpetuación de seguridad. (...) El nuevo Reglamento Básico será el instrumento normativo en el que se deberá encuadrar la sustitución de las prácticas manicomiales que subsisten al interior de los Hospitales, así como la formalización de los nuevos dispositivos de asistencia intra y extra institucionales. (...) Cada Hospital interdisciplinario deberá abocarse a viabilizar la externación no expulsiva de los pacientes institucionalizados y la implementación de dispositivos sustitutivos de las lógicas manicomiales. (...) se entiende por Servicios Alternativos todos aquellos dispositivos institucionales y comunitarios, clínicos, productivos, educativos y culturales que se orienten al sostenimiento de prácticas de asistencia al sufrimiento psíquico, que no sean expulsivas ni generen exclusión ni encierro de las personas, no atenten contra sus derechos humanos y permitan el desarrollo de sus capacidades y su inclusión en el ámbito comunitario". (LPSM N¹0.772, B.O., Santa Fe, Argentina, 25/12/1991 y D.R. 2155/2007)

Entre ellos ubica a dos dispositivos que nos interesan particularmente para el presente trabajo: Casa de Medio Camino y Casa de Medio Camino bajo la modalidad de Viviendas Asistidas.

Posteriormente, en el 2010, en el artículo $11^{\circ}$, la LNSM N² 26.657 enunciará:

"La Autoridad de Aplicación debe promover que las autoridades de salud de cada jurisdicción, en coordinación con las áreas de educación, desarrollo social, trabajo y otras que correspondan, implementen acciones de inclusión social, laboral y de atención en salud mental comunitaria. Se debe promover el desarrollo de dispositivos tales como: consultas ambulatorias; servicios de inclusión socialy laboral para personas después del alta institucional; (...) prestaciones tales como casas de convivencia, hospitales de día, cooperativas de trabajo, centros de capacitación socio-laboral, emprendimientos sociales, hogares y familias sustitutas".

Acerca del artículo citado, el Decreto 603/2013 reglamenta:

"Facúltese a la Autoridad de Aplicación a disponer la promoción de otros dispositivos adecuados a la Ley $\mathrm{N}^{\circ}$ 26.657 (...) dispositivos habitacionales y laborales con distintos niveles de apoyo, atención ambulatoria, sistemas de apoyo y atención domiciliaria, familiar y comunitaria en articulación con redes intersectoriales y sociales, para satisfacer las necesidades de promoción, prevención, tratamiento y rehabilitación, que favorezca la inclusión social". (D.R. 603/2013 de la Ley No 26.657, reglamentación artículo $11^{\circ}$ )

En consonancia, el Ministerio de Salud de la provincia de Santa Fe, mediante la Resolución $\mathrm{N}^{\circ}$ 2148/2013, formaliza como dispositivo de salud alternativo a las lógicas manicomiales las "Residencias Compartidas para Usuarios de Salud Mental". Entre sus consideraciones, caben mencionar:

"Que tanto la normativa nacional como la Ley Provincial No 10.772 rezan que, en el proceso de atención de la salud mental deberán 
privilegiarse el ámbito extrahospitalario, las alternativas que menos restrinjan la libertad de los pacientes y la conservación de sus lazos familiares y sociales" -artículo $9^{\circ}$ de la LNSM $\mathrm{N}^{\mathrm{o}} 26.657$ y segundo párrafo artículo $1^{\circ}$, de la Ley Provincial No 10.772-;

"Que ambas leyes propugnan la sustitución de las lógicas manicomiales y el reforzamiento de dispositivos alternativos que tiendan a la inclusión social, laboral y la atención en la salud mental comunitaria, mencionando entre dichos dispositivos a las "casas de convivencia" -artículo $11^{\circ}$ de la Ley Nacional $\mathrm{N}^{\circ}$ 26.657-, así como las "Casas de Medio Camino: Destinadas para aquellos pacientes estabilizados con niveles de autonomía que no requieren asistencia específica" y las "Casas de Medio Camino bajo la modalidad de Vivienda Asistidas: Destinadas para pacientes estabilizados con un nivel de dependencia cuya asistencia requiera sostenimiento específico con un apocamiento mínimo" -artículo $18^{\circ}$, incisos i) y j), de la reglamentación provincial-.

\section{Antecedentes de la Casa Asistida de Mujeres}

Es desde el marco de la citada Ley Provincial $\mathrm{N}^{\circ} 10.772$ (1991) y su reglamentación (2007) que se impulsó la transformación del Centro Regional de Salud Mental provincial y la creación de los nuevos dispositivos sustitutivos de las lógicas manicomiales. La primera Casa Asistida de Mujeres comenzó a funcionar en junio de 2009, con cuatro mujeres residentes coordinadas por un equipo interdisciplinario.

En el proceso de constitución de la Casa Asistida, fue clave la participación de los actores del hospital monovalente quienes contribuyeron a la paulatina transformación que derivó en la creación del dispositivo. Ello estuvo dado por el sostenimiento desde las diferentes autoridades del C.R.S.M. y grupos de trabajadores, de la propuesta de desinstitucionalización de los pacientes de larga internación. En las gestiones de los últimos quince años, a pesar de las resistencias de los trabajadores ante los nuevos cambios, se fueron dando modificaciones tales como la movilización de los médicos y su redistribución desde el sector de internación hacia los consultorios externos; la transformación de los equipos de internación y su constitución en interdisciplinarios conformados por psiquiatras, psicólogos, trabajadores sociales y enfermeros. La incorporación de abogados a los dispositivos del hospital fue otro importante logro.

En este contexto, trabajadores del hospital realizaron una investigación (2005) con respecto a los pacientes hospitalizados, en cuanto a su situación y el tiempo que llevaban en la institución. Para ello propusieron dos categorías de análisis: pacientes de larga internación/institucionalización, es decir los llamados crónicos; y pacientes revoolving door o puerta giratoria, los que durante el año tenían varias internaciones. La investigación reveló que uno de los principales problemas que tienen los pacientes de larga institucionalización era la falta de vivienda o la imposibilidad de vivir con algún familiar. A eso se le sumaba las situaciones de desempleo.

\section{Procesos de su constitución}

A partir del material de las entrevistas ${ }^{5}$ realizadas a los distintos actores involucrados,

\footnotetext{
${ }^{5}$ Las entrevistas referidas en este artículo fueron realizadas en el marco de la investigación exploratoria del cual el TIF es resultado. Serán diferenciadas sólo por el año de producción para preservar el anonimato de los actores, las mismas fueron realizadas en los siguientes períodos: marzo y noviembre de 2012; marzo y octubre de 2013; y enero de 2014.
} 
efectuaremos una aproximación a la reconstrucción de los principales hitos del proceso de constitución de esta Casa de Medio Camino en su modalidad de Vivienda Asistida de Mujeres.

Una de las autoridades entrevistadas reseña:

\begin{abstract}
"Necesitábamos casas asistidas porque había una población crónica que no podía volver a sus hogares, la mayoría de los dispositivos estaban muy pegados a la internación. Ya había experiencias de usuarios que vivían en pensiones, no eran muchos $(. .$.$) no había otra$ alternativa más que las pensiones, sin embargo, había personas que no podían vivir en ellas”. (Entrevista, 2013)
\end{abstract}

Una de las coordinadoras reflexiona:

\begin{abstract}
"Como trabajadoras dependemos económicamente del Hospital. El alquiler de la casa fue gestionado desde el mismo, como también los alimentos, los artículos de limpieza. Los pacientes eran del hospital, la casa depende de la dirección del C.R.S.M., pasa luego a hacerse cargo directamente de estos dispositivos la Dirección Provincial de Salud Mental en cuanto al alquiler, los alimentos, los artículos de limpieza, lo necesario para el funcionamiento". (Entrevista, 2013)
\end{abstract}

Desde el hospital estaba fuertemente instalada la idea de concretar dispositivos de residencias compartidas como sustitutivos a las lógicas manicomiales. ¿A partir del alquiler de la casa, qué movimientos se empezaron a realizar en el hospital?

Como claramente expresan los distintos actores entrevistados, se empieza a reflexio- nar sobre la posibilidad de llevar a ese espacio, uno de los talleres de producción que funcionaba en el Centro Cultural del hospital, toma relevancia la idea de empezar a habitar la casa a través del taller, del trabajo, que las pacientes hubiesen hecho lazos; además de elegir al personal y pensar en la selección de las moradoras, que, hasta ese momento, seguían siendo pacientes internadas en los pabellones.

Por un lado, ya contaban con la casa, pero todavía faltaba terminarla de acondicionar. Por otro lado, cuando se alquiló la casa se tuvo en cuenta que tuviera un espacio grande para trasladar el taller de producción de costura: "para las mujeres que viven ahí el taller es un espacio que sigue siendo un soporte fundamental en cuanto a su cotidianidad”. (Entrevista, 2013)

¿Cómo se fue dando la selección de las moradoras?

"Desde los que estábamos pensando el nuevo dispositivo, aparece la pregunta: ¿a quién había que definir primero, a los pacientes o a los que debían acompañarlos? Se hizo un relevamiento de los pacientes de larga internación y que estuvieran en condiciones de externarse. Y se empezó a trabajar con ellos acerca de trasladarse a la Casa, aparecieron temores, dudas y entusiasmo (...) En sí lo primero que hicimos fue reflexionar cuestiones con los compañeros del hospital, principalmente queríamos que entendieran lo que estábamos haciendo (...) y es así que se puso en movimiento algo muy interesante, todos empezaron a apropiarse y a participar de la nueva iniciativa. La casa estaba vacía, había que amoblarla y no contábamos con presupuesto suficiente. Fueron los compañeros los que donaron mesas, sillas, sábanas, etc.” (Entrevista, 2103) 
Esto permite indagar acerca del proceso que realizaron los diferentes actores - directivos, coordinadores, trabajadores del hospital- implicados en el nuevo dispositivo, evidenciando la puesta en marcha de un proceso de reflexión acerca de sus propias prácticas y en particular con respecto a la Casa Asistida. Dicho proceso conlleva a un estado de interrogación permanente entre el modo de atención que se da adentro del hospital y el modo de atención que se da por fuera. En relación a: ¿que implica asistir a estas mujeres? Los equipos interdisciplinarios empiezan a perfilar la idea de la asistencia como un cuidado, pero no como un único modo esquemático de trabajar con las residentes de la casa asistida, como era en el hospital- horarios para las actividades de la vida diaria, tales como alimentación, higiene, descanso, etc.- sino pensar la asistencia en relación a las singularidades de las moradoras a través de estrategias terapéuticas complejas y singularizadas.

Es así que, en las Jornadas realizadas sobre estos dispositivos, aparecieron ciertas interpelaciones con respecto a los modos de intervención manicomiales tales como: "rigidización de respuestas ante una variedad de sufrimientos", "pérdida de la autonomía de los internados", tratar al "sujeto como un objeto". Así una de las disertantes expone: "Encierro, exclusión y ocultamiento al parecer esto cuando trabajábamos allí (hospital) es tan ajeno a nosotros y esas lógicas institucionales y manicomiales construyen subjetividades". (Córdoba, S. 2013)

La discusión en esas jornadas gira alrededor del señalamiento de la importancia del sostenimiento de intervenciones clínicas singularidades en relación a los usuarios que viven en estos dispositivos, para no caer en el riesgo de que aquello que sustituye al manicomio, pueda tener también un efecto rígido y devastador como lo fue en su momento el manicomio mismo, ya que la invisibilidad de ciertas prácticas y las lógicas pueden ser manicomiales más allá del manicomio.

\section{Asistencia.}

Los equipos de trabajadores que acompañaron el sostenimiento y funcionamiento del dispositivo acuerdan acerca de lo que implica la asistencia terapéutica de las moradoras, que complejiza el camino a transitar.

"No es una asistencia al modo del Hospital, pero tampoco pueden vivir solas. Asistencia en el sentido de acompañarlas en que puedan continuar con sus quehaceres, hacer su comida, que hagan actividades que tengan que ver con sus deseos. (...) Es en esta lógica de trabajo que la palabra asistencia tiene que ser pensado como presencia (...) por lo menos esta asistencia, este cuidado, esta presencia tiene sentido que en el momento de acompañar, este acompañamiento tiene que ser pensado como una marca, como una fuente de significación, como algo que se construye colectivamente, que ante la no presencia nuestra, las mujeres puedan seguirlo. Es por esto que cuando me preguntan que es la asistencia digo que es presencia en la ausencia, continuidad en la discontinuidad. En esta forma de trabajo, en una casa donde uno va, estás unas horas y luego te retiras, las mujeres se acompañan entre ellas y tienen que recuperar en determinados momentos esto que hemos construido como rutas posibles, como puentes/fuentes de significación para transitar determinados momentos del otro día. (Entrevista, 2013) 
Externación ¿En qué consistió el proceso de externación de estas cuatro mujeres? Fueron, entre otros, las preguntas que se indagaron al momento de realizar los encuentros con los actores implicados. Una de ellas va marcado coordenadas de este proceso en su relato:

"Al principio estaba todo el tiempo con ellas. Fue un proceso paulatino, comenzamos a salir del hospital, a transitar en la ciudad, en colectivo. Eran otros tiempos, cuarenta y cinco minutos en el cole, en relación al tiempo "muerto" en el hospital. Aparecieron dificultades singulares de viajar en colectivo, disparó en ellas las más diversas fantasías, miedos, persecuciones: temores ante la mirada de los otros, de creer que el chofer podía chocar, etc. (...) Viajar, llegar a la casa, estar en la casa, armar los espacios amueblándolos. Fueron apropiándose del modo de habitar una casa, transitar el tiempo y el espacio, por ejemplo, tomando un mate, ir a limpiarla. Cuando llegaban donaciones, entre ellas había cosas rotas, el trabajo consistía en que eligiesen lo que a ellas les gustaba, que no debían aceptar pasivamente todo lo que allí llegaba”. (Entrevista, 2013)

Espacio y tiempo. Las instancias de trabajo clínico con las moradoras, en relación a la construcción de las categorías de espacio y tiempo adquirieron ciertas particularidades.

"Luego empezamos a ir en otro momento del día, a la tarde, proceso que llevó seis meses aproximadamente. Recuerdo que en la primera casa que se alquiló las puertas de algunas piezas no cerraban, además de arreglarlas, se trabajó sobre la importancia de que haya espacios delimitados. También cada vez que íbamos, otro tema que surgía era el de las llaves, el de asegurarse de que la puerta estuviera cerrada. Las moradoras volvian a tener una llave, a diferencia del hospital, donde las puertas permanecen abiertas o son otros los encargados de cerrarlas, controlarlas (...) Otro momento para elaborar fue lo relacionado al fin de semana, que pasaba en el barrio, cuáles eran los momentos de ocio, qué actividades se les ocurría que podían realizar. Así se fue instituyendo los paseos a la plaza (...) Con respecto al teléfono, en el hospital, cuando se les permitía llamar, esto era en algunos horarios, de tal hora a tal otra, y con la modalidad de la llamada del hospital, que a los tres minutos se corta la comunicación. Acá en la casa no, entonces la situación que se planteó era como entrar en una regulación pero que no sea una norma desde lo manicomial'. (Entrevista, 2012)

\section{Las moradoras de la Casa Asistida}

Antes que presentemos a las moradoras volvamos la mirada una vez más hacia la institución elegida para el recorte del problema que este trabajo aborda, y de la cual estas mujeres se externaron. Como describíamos anteriormente el C.R.S.M. es un hospital público monovalente con internación en salud mental y guardia permanente. Como hospital público está principalmente destinado a la atención y prestación de servicios de salud mental para personas de bajo recursos económicos. Además de la atención de pacientes en crisis, a través de la guardia psiquiátrica permanente de $24 \mathrm{hs}$ y la atención de pacientes crónicos, cuenta también: con atención ambulatoria en consultorios externos, atención domiciliaria, 
talleres culturales y dispositivos de admisión. Todas estas instancias son desarrolladas por equipos interdisciplinarios.

Ediliciamente se divide en cuatro bloques. La parte de adelante está destinada al funcionamiento de la dirección, administración, consultorios externos, farmacia y oficina de abogados. Separados del resto a través de una puerta, que comunica con el patio, y es por donde se accede a los dos pabellones de internación cada uno de dos plantas, cuatro salas, baños de uso común, office de enfermería y consultorios de atención. Hacia el lado opuesto y paralelo a los consultorios externos se levanta el edificio o parte correspondiente a la guardia que tiene entrada por otra calle de la ciudad. Otra parte de la estructura está destinada al comedor y a los talleres culturales. Todas las estructuras edilicias se comunican internamente a través de un patio, con puertas que están bajo llaves (la que da al sector de guardias) y hay un guardia que controla la salida al exterior a través del pabellón del frente.

En dicho pabellón de entada correspondiente a los consultorios externos y dependencias administrativas podemos observar un movimiento casi frenético de personas que van y vienen, un colectivo heterogéneo que sin embargo comparte algunas cualidades y se diferencia claramente de otras personas que también están por allí. El grupo semoviente está compuesto por profesionales, residentes de psiquiatría, administrativos, porteros y algún que otro personal de servicios generales y vigilancia. Todos parecen muy atareados y apurados. El otro grupo está constituido por los pacientes que esperan para ser atendidos por su psiquiatra o psicólogo, o para sacar un turno. En su mayoría están quietos y cabizbajos, otros simplemente están como ausentes, pero a todos parece identificarlos la quietud y la espera.

Para acceder a los pabellones de interna- ción lo realizamos a través del patio, antes de que la persona de seguridad abra con su llavín. El patio es amplio, la mayor parte con piso de material y con algunos árboles grandes. Además, hay varias mesas de cemento con sus correspondientes bancos, y en algunas de ellas hay pacientes compartiendo una mateada o charlando. También hay otros en silencio o escuchando la radio, y un par están tirados como durmiendo una siesta, descalzos y con el torso desnudo.

Si bien el hospital fue modificándose y cambiando sus prácticas en función de las nuevas políticas de desmanicomialización sigue siendo un hospital y no un lugar para residir.

¡Qué distinto fue encontrarnos con dos de las moradoras de la Casa Asistida, a la salida de un supermercado y no en el patio o pabellón del hospital del cual partieron!

Mientras van terminando de hacer las compras, se puede observar: una de ellas chequea el ticket, la otra menciona a la cajera la dirección donde debía ser enviada la mercadería. En un bar se realizaron las entrevistas (enero 2014). Están alegres, interesadas en que conversáramos. Mientras guardan los tickets y la calculadora, comentan: "qué caro que está todo, desde la última vez que vinimos aumentaron los productos".

Luego describen sobre los momentos de sus crisis subjetivas y sus internaciones y la gran diferencia de lo que realizan en la actualidad: una de ellas se ocupa de la contabilidad de los gastos de la casa y del taller de producción de costura. Detallan sobre las diferentes ayudas económicas que reciben tales como: planes promover, cupones o vales para mercadería y tarjetas de ciudadanía, ingresos con los que se desenvuelven para cubrir algunos de sus gastos. Una de ellas calcula el importe de esos beneficios y en qué los distribuyen.

Posteriormente pasan a narrar sobre su 
cotidianidad en la casa, una ayuda en el preparado de la comida, limpia, la otra moradora se ocupa de las plantas. Se despiertan más bien temprano: "compartimos unos mates mientras cuchicheamos en la cocina de todo, de lo qué vamos a preparar para comer, de los hijos". Aclaran que antes, estando en el hospital no hablaban entre ellas, "ni con nadie".

Sobre la casa dicen: "es muy linda tiene dos dormitorios, un living comedor, cocina y baños grandes. Un espacio donde realizamos yoga y un jardín con muchas plantas". "Estamos mejor que en el hospital" Ambas coinciden en cuanto al tiempo libre: "realizamos más actividades", "podemos bañarnos cuando queremos, lavarnos nuestra ropa". "Para hacer nuestras cosas y recibir visitas. En cambio, en el hospital está todo programado, la medicación, la hora en que te tenés que bañar. Ahora nos dejan medicación por un mes y nosotros las tomamos solas".

Asimismo, mencionan que estando en la Casa Asistida vuelven a retomar proyectos de antaño tales como continuar estudios interrumpidos.

Al hospital lo asocian con aburrimiento y tristeza, cuando presenciaban las crisis de los otros internos, por más que contaran con espacios de producción y equipos de profesionales que las atendían.

Una moradora explica que durante su internación que se extendió a tres años trajo como consecuencia que los lazos con sus hijos se fuesen deteriorando: "allá no iban a verme", entonces con el acompañamiento de las coordinadoras empezaron a generase esos encuentros, "al principio era en un espacio público, después los pasaba a buscar y juntos íbamos hasta la Casa Asistida, hasta que finalmente comenzaron a venir por su cuenta".

En relación al dispositivo casa asistida, una de las moradoras dice: "me tomó por sorpresa, no sabía de su existencia”. El ma- yor temor que en aquel entonces le producía el nuevo cambio, era la convivencia con otras personas. "Al principio no hablábamos ni entre nosotras, en el hospital no nos conocíamos porque estábamos en diferentes pabellones". También menciona como diferencia: "en el hospital para ver tele era un problema, porque los otros internos iban y te cambiaban de canal'. Recuerda que cuando fueron a compartir la fiesta de fin de año que se realiza en el C.R.S.M. vieron a sus ex compañeros "deteriorados" y "crónicos".

En cuanto a la atención de su salud, en la actualidad recurren al Hospital General, emplazado en el barrio, en particular para la realización de los tratamientos psiquiátricos y psicológicos, esto fue un proceso paulatino, ya que, en los primeros tiempos, continuaban con la atención por consultorios externos con los mismos profesionales que la asistían durante la internación. El acceder al hospital cercano a su vivienda les permitió también empezar a empoderarse e implicarse activamente en la gestión y sostenimiento de la importancia de los controles ginecológicos, nutricionales, endocrinológicos, cardiológicos, etc.

En cuanto a sus actividades productivas, tres veces a la semana participan de un taller de producción de costura: bordando o cociendo o remallando. Y una vez por semana participan de una feria donde ofrecen los productos que confeccionan. En cuanto a las actividades recreativas, los sábados van un bar o heladería.

Con la tercera moradora el encuentro fue realizado en el comedor de la Casa Asistida, orgullosa comenta el hecho de haber podido comprarse un somier y televisor para su habitación y haberla podido adornar con objetos personales como cuadros y fotos. La habitación la comparte con otra de las residentes quien la ayuda con algunas cuestiones ya que 
presenta dificultades en su movilidad. Para poder trasladarse por la ciudad, cuenta con el sostén de un acompañante terapéutico.

La cuarta moradora que ingresó con posterioridad a las tres primeras, describe que al principio solo se quedaba los fines de semanas y paulatinamente fue incorporando mayor cantidad de días logrando luego su residencia permanente. También describe que en la casa cuenta con un lugar privado para que su hijo la visite. De los recuerdos del hospital dice: "es un lugar feo, algunos de internos se peleaban, la ropa que tenías para usar no siempre te quedaba bien y muchas veces perdía mis objetos personales".

Es muy significativa la expresión que utilizan para denominar el lugar donde habitan: es nuestra propia casa.

\section{Análisis. Reflexiones finales}

Se entiende como anti manicomiales a aquellas lógicas implícitas en diversas prácticas, ya sean jurídicas, médicas, psicológicas, económicas, políticas, que van en contra de la medicalización en exceso, el silenciamiento y la exclusión social de los padecimientos psíquicos. Lógicas manicomiales que separa un objeto ficticio, la enfermedad, de la integralidad de las personas y del tejido histórico-social en el que advinieron como tales y en el que surgieron sus problemas.

En consecuencia, pensar la desinstitucionalización es hacer referencia a la tarea de deconstruir esas producciones institucionales existentes y constitutivas de los imaginarios culturales compartidos. Esas producciones, si bien pueden ir más allá de los manicomios reales, encuentran en sus lógicas derivadas, su fuente y su consistencia. Por lo tanto, la deconstrucción coincide con la creación y sostenimiento de experiencias sustitutivas que se van convirtiendo en dispositivos e instituciones nuevas $y$, por ende, en constante transformación.

Retomando las propuestas conceptuales italianas en relación al departamento terapéutico, los autores, al pensar en la rehabilitación, diferencian "casa" de "habitar". Mientras que por casa se entiende simplemente un lugar para vivir, sin ser necesario recurrir a habilidades donde sólo será suficiente con "estar"; en cambio "habitar" representa algo más complejo y diverso: "adquirir habilidad contractual, ejercer un poder material o simbólico", ser partícipes y protagonistas en cuanto se esté viviendo". Y agregan que el manicomio es el símbolo por excelencia del "no habitar", más bien del "estar" (2013 Ricerca Dipartimento interaziendale ASL5. Collegno/ASO S.Luigi Gonzaga Universitario di Salute Mentale - Proposte di linee guida per i gruppi appartamenti, diretto dal Prof. Pier Maria Furlan)

Asimismo plantean: "Por cierto, sería necesario apuntar a construir habitaciones pensadas como lugares de transición hacia el habitar verdadero, entender estas estructuras como recorridos donde se devuelva una parte de aquellas especificidades que caracterizan una casa; de manera que la persona adquiera competencias y capacidades para habitar, es decir la discrecionalidad, el poder de decidir y el poder contractual".

Si consideramos el planteo de Basaglia (1972), la regla institucional tiende a destruir al individuo para perpetuarse, asegurando su propia supervivencia. La institución tiende entonces a asumir el rol de custodio confirmando con el tiempo el aspecto crónico de la enfermedad. Sin desconocer el profundo planteo de Basaglia, hay que tener en cuenta que rehabilitar implica afrontar un proceso recíproco en el que interviene el terapeuta, la persona con padecimiento psíquico y la comunidad; por lo tanto, la intervención tiende 
a influir en ámbitos más generales como: la búsqueda del nivel más alto posible de autonomía y de la libertad de expresión del sujeto, acompañar a la persona con respecto al logro de la modificación de vínculos que impliquen modos y relaciones rígidas que hacen obstáculos en sus lazos sociales y promover en el contexto la inserción de las personas que sufren. Estos objetivos deben ser incluidos en un proyecto integral de vida, que no puede ser ni predeterminado, ni decidido sin tener presente la singularidad del sujeto.

Para el presente análisis es enriquecedor poder comparar el dispositivo de Casa Asistida con la propuesta italiana de los departamentos terapéuticos en tanto representan una estructura intermedia en el ámbito de la asistencia de la salud mental. Se configuran como lugares adecuados para el desarrollo de intervenciones clínico terapéuticas orientadas a la recuperación de la dimensión del tiempo, del espacio y de las relaciones en el plano de las vivencias singulares. Trabajar sobre la cotidianeidad y sobre el hecho de habitar un lugar, implica necesariamente "el encuentro" donde se sitúan las residencias.

Es desde aquí que cobra relevancia el sentido de "habitar" en un lugar del que los usuarios se puedan apropiar, es decir, contar con el poder de utilizar un objeto (habitación y sus recursos) y de prohibir que los demás lo utilicen, en lugar de "vivir en", en el sentido de simplemente "estar". La ausencia de posesión implica la ausencia de autorregulación, es decir la ausencia de poder, de perseguir los propios propósitos en las formas y tiempos establecidos singular y autónomamente.

En otras palabras, el habitar, el estar juntos, el "vivir con" y crecer con, representan un potencial terapéutico que responde junto a las características de residencias de reducidas dimensiones, a la necesidad de seguridad de integración y de afectos que es propio de una casa: un objeto estable, un punto de seguridad que recibe el usuario y lo ubica en un espacio físico. La vida cotidiana, compartir un lugar, el espacio y el tiempo se centra sobre la acción en lugar de la verbalización; se comparten experiencias de todos los días en una relación emocional y física muy íntima, cada gesto se comparte y se valoriza.

En relación a esto, el aporte de la experiencia italiana nos permite reflexionar sobre la importancia de que, tanto moradores como el personal del equipo, conozcan y construyan la distinción entre espacios privados y comunes dentro de la casa. Es necesario, también, que el dispositivo se configure como un lugar de habitación civil que favorezca los objetivos de inclusión social y autonomía para lo que es fundamental que se sitúe en un lugar no aislado para que los servicios de base sean de fácil acceso.

Es importante destacar que, en nuestra región, si bien se promueve el desarrollo de dispositivos como las casas de convivencia, en tanto servicios cuya meta es la inserción social de los pacientes que han padecido pérdidas $\mathrm{y}$ mortificaciones en su yo como consecuencias de la institucionalización en instituciones totales, no se puede soslayar que las creaciones de estos dispositivos están en una etapa inicial en función de lo propuesto por la ley. La Casa de C.R.S.M así lo revela, y no por el hecho de que no haya logrado la desinstitucionalización de sus moradoras y el restablecimiento de sus derechos, es notorio el compromiso e implicación de este grupo de trabajadores, sino que son experiencias que recién van logrando cierta articulación y afianzamiento con otros servicios. Es claro que esta experiencia adquirirá otro sentido en fase de consolidación del sistema de salud mental y de la constitución de tantos otros servicios.

La Casa Asistida ha conseguido devolver aquello que en su momento se les profanó 
a estas cuatro mujeres. Pero la realidad indica que son sólo cuatro. Entonces, ¿cómo prosigue esta experiencia? ¿Se cuenta con un plan de externación desde el hospital monovalente?

Cuando los departamentos o casas ya no sean protegidas o asistidas, es decir, cuando se avance en la inclusión social de estos pacientes, los interrogantes de la experiencia francesa, nos permiten vislumbrar las situaciones que pueden darse en la comunidad, y a las que habrá que aportar soluciones que en muchos casos abrirán otras preguntas resultado de la complejidad abordada. Acudimos a ese material ya que sitúa la cuestión de las crisis subjetivas en la comunidad. ¿De qué manera se prepara nuestra región para esto? Si bien será necesario estar atentos, no hay dudas de que la Ley Nacional de Salud Mental traslada, lo que antes se recluía y separaba en un acto de segregación al hospital monovalente, al corazón de la comunidad.

\section{Referencias}

- Alberdi, J., Coll, L., Mutazzi E. \& Vismara E. (2005) La problemática de la institucionalización crónica y el fenómeno de "revolving door" en pacientes usuarios del Centro Regional de Salud Mental "Dr. Agudo Ávila”. Revista Cátedra Paralela. Argentina.

- Amarante, P. (2009) Superar el Manicomio: Salud Mental y atención Psicosocial. Buenos Aires, Topia Editorial.

- Basaglia, F. (1972) La institución negada. Informe de un Hospital Psiquiátrico. Barcelona, Barral Editores.

- Basaglia F. (1979) La condena de ser loco y pobre. Alternativas al manicomio. Buenos Aires, Editorial Topia, 2009.

- Centro de Estudios Legales y Sociales \&
Mental Disability Rights International (2007) Vidas Arrasadas. La segregación de las personas en los asilos psiquiátricos argentinos. Un informe sobre derechos humanos y salud mental en Argentina.

- Córdoba, S; Scandizzi, A. (2013). Casas Asistidas de Mujeres. Ponencia presentada en Jornadas sobre Dispositivos de Sustitución Manicomial: Casas Asistidas, Casas compartidas y otros dispositivos habitacionales", realizada por departamento de docencia e investigación del C.R.S.M. "Dr. Agudo Ávila", Rosario, Argentina.

- Cuneo, C. (2013) Seminario Procesos de desinstitucionalización en Carrera de Especialización en Psicología Clínica Institucional y Comunitaria. Facultad de Psicología. Universidad Nacional de Rosario, Argentina.

- Decreto reglamentario $\mathrm{N}^{\circ}$ 2155/2007 de la Ley Provincial de Salud Mental $N^{\circ}$ 10.772, Boletín Oficial de la provincia de Santa Fe. Santa Fe, Argentina (28 de setiembre de 2007).

- Decreto reglamentario $\mathrm{N}^{\circ}$ 603/2013 de la Ley Nacional de Salud Mental 26.657. Boletín Oficial. Buenos Aires, Argentina (29 de mayo de 2013).

- Dell' Acqua, P. (2011) "Desde el manicomio hacia los servicios de salud mental en el territorio" en Ideass Italia Innovación para el Desarrollo y la Cooperación Sur-Sur (www. ideassonline.org).

- Galende, E. Editorial en Revista Salud Mentaly Comunidad, Ediciones de la UNLa. (29 de setiembre 2011).

- Genot-Pok I., Charbonnel, A., De LardHuchet, B., et al. Réforme des soins psychiatriques: voir clair dans une réforme encore obscure. En Actualités jurisanté (CNEH), N ${ }^{\circ}$ 76, 2011/12. - 3-30

- Guigue, S. Présentation de la loi relative aux droits et à la protection des personnes faisant l'objet de soins psychiatriques 
et aux modalités de leur prise en charge. En Revue Droit \& Santé, n45, 2012/01, p. 47-53

- Goffman, E. (1972), Internados. Ensayos sobre la situación social de los enfermos mentales. Buenos Aires, Amorrortu Editores.

- Instituto de Derecho e Integración. Colegio de Escribanos de la Provincia de Santa Fe. Revista N5, año 3 (2011)

- L'accès et le maintien dans le logement des personnes ayant des difficultés de santé mentale - L'union sociale pour l'habitat. Les Revues. Paris, France. 2006 (www.unionhabitat.org)

- Käes R, Bleger, J. Fornari, F. Fustier, P. Roussillon, R. \& Vidal, J.P. (1998) La institución y las Instituciones, Argentina, Ed. Paidós.

- Hermosilla, A. \& Cataldo, R. "Ley de Salud Mental 26.657. Antecedentes y Perspectivas" en Psiencia Revista Latinoamericana de Ciencia Psicológica. Volumen 4, nro. 2 (noviembre 2012), p. 134 a 140.

- Ley No 10.772 Ley Provincial de Salud Mental. Boletín Oficial. Santa Fe, Argentina (25 de diciembre de 1991).

- Ley No26.657 Ley Nacional de Salud Mental. Boletín Oficial N 32041, Buenos Aires, Argentina (3 de diciembre de 2010).
- Resolución No 2177. Plan Nacional de Salud Mental. Boletín oficial del Ministerio de Salud de la Nación. Buenos Aires, Argentina (8 de octubre de 2013).

- Resolución $N^{\circ}$ 2148. Boletín Oficial de la Provincia de Santa Fe, Santa Fe, Argentina. (22 de octubre de 2013)

- Ricerca Dipartimento interaziendale ASL5. Colle/ASO S.Luigi Gonzaga Universitario di Salute Mentale (2013) - Proposte di linee guida per $i$ gruppi appartamenti, diretto dal Prof. Pier Maria Furlan. Italia

- Resolución N²148. Boletín Oficial de la Provincia de Santa Fe, Argentina. 22 de octubre de 2013.

- Saidon, O y Troianovki, P (Comp.) 1991. Politicas en Salud Mental. Buenos Aires, Lugar Editorial.

- Salud Pública y Salud Mental: el nacimiento de la política del sector. En Rev. Asoc. Esp. Neuropsiq. - Vol. XI. No 37. Año 1991.

- Scandizzi, A (2013). Casa Asistida C.R.S.M "Dr. Agudo Ávila". Ponencia presentada en el III Congreso de Salud Pública de la Provincia de Santa Fe, Rosario, Argentina.

- Wagner Sousa Campos, G. (2001) Gestión en salud. En defensa de la vida. Buenos Aires, Caribe- Editare. 